\title{
THE ASSOCIATION OF PERIODONTAL DISEASES WITH TYPE 2 DIABETES MELLITUS AND DIABETES LINKED DYSLIPIDEMIA IN PAKISTANI POPULATION
}

\author{
Zara Khalid Khan, Muhammad Uzair Khan*, Amna Khalid Khan**, Rukhsana Muttee***, Rubeena Khalid**, \\ Malik Muhammad Khalid Bashir Khan** \\ Army Medical College/National University of Medical Sciences (NUMS) Rawalpindi Pakistan, *City University Peshawar Pakistan,

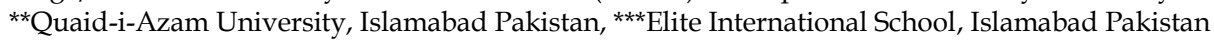

\begin{abstract}
Objective: To determine the development of dyslipidemia in type 2 diabetics with and without periodontal diseases.

Study Design: Comparative cross-sectional study.

Place and Duration of Study: Fauji Foundation Hospital and Pak Emirates Military Hospital, Rawalpindi, Pakistan, from Jan 2018 to Jan 2019.

Methodology: Total number of participated subjects (males and females) was 75, with age ranging from 23 to 72 years. Their tests included blood sugar, lipid profile, HbA1c and hepatitis screening. Oral health parameters were divided into two categories; type 2 diabetes mellitus with and without periodontitis.

Results: Out of 75 patients, males and females were 19 (25.3\%) and 56 (74.7\%). Females with periodontitis 20 $(74.1 \%)$ and without periodontitis $36(75 \%)$ possessed larger numbers. Mean ages [56.33 \pm 10.88 years] had a higher incline, along with significantly altered total cholesterol [5.41 $\pm 1.70 \mathrm{mmol} / \mathrm{l}]$, triglycerides $[2.55 \pm 1.46$ $\mathrm{mmol} / \mathrm{l}]$, low and high density lipoproteins [3.01 $\pm 1.22 \mathrm{mmol} / \mathrm{l}, 0.97 \pm 0.48 \mathrm{mmol} / \mathrm{l}]$ in type 2 diabetes mellitus with periodontitis. Patients of diabetes without periodontitis showed mild derangements for triglycerides [1.88 \pm $1.22 \mathrm{mmol} / \mathrm{l}$. Patients of diabetes with dyslipidemia and periodontitis 16 (59.3\%) were documented highest in number, compared to other type 2 diabetes mellitus groups.

Conclusion: There was an overall significant association between type 2 diabetes mellitus related dyslipidemia and periodontitis. Also, significant difference in age and gender of study subjects was noted that affected middle aged females more often than middle to late aged males.
\end{abstract}

Keywords: Age, Dyslipidemia, Gender, Oral health, Periodontal diseases, Type 2 diabetes mellitus.

This is an Open Access article distributed under the terms of the Creative Commons Attribution License (http://creativecommons.org/licenses/by/4.0), which permits unrestricted use, distribution, and reproduction in any medium, provided the original work is properly cited.

\section{INTRODUCTION}

The diabetes mellitus $(\mathrm{DM})$ is one of the $21^{\text {st }}$ century's non communicable concocted metabolic disorder, generated due to the insulin (generation, utilization or combined) problems, characterized by the raised glucose levels of blood, and is spreading too fast, creating colossal endangerments ${ }^{1,2}$. Out of all the three types of DM (type 1, type 2 and gestational), the most common is type 2 (T2DM), that reports nearby $90-95 \%$ of all the diagnosed scenarios. The prevalence of DM across the globe includes population of 415 million, which is estimated to incline to 642 million in the near future, according to the International Diabetes Federation (IDF) ${ }^{3}$. There is very high

Correspondence: Dr Zara Khalid Khan, Dept of Biochemistry \& Molecular Biology, AM College, Rawalpindi Pakistan

Received: 05 May 2020; revised received: 15 Sep 2020; accepted: 17 Sep 2020 prevalence of DM with its associated complications and risk factors in the Pakistani population as well. According to the WHO fact sheet, DM is $13 \%$ (7.1 million) prevalent in the country in both genders, affecting $17.15 \%$ of the rural and $22.04 \%$ of the urban areas ${ }^{4}$. Dyslipidemia as a complication of T2DM is getting massive prevalence in Pakistan, triggering atherosclerosis and many other health risks 5 .

Oral health is a significant component of the systemic health, that plays an essential role in the maintenance of overall good health and wellbeing, which if not conserved can trigger worse circumstances. Also, DM has been reported to cause some noxious effects on the oral health of patients. Dyslipidemia and altered glucose metabolism in T2DM have shown positive associations with multiple periodontal and oral inflam- 
matory diseases, owing certain roles to proinflammatory cytokines for inducing such hyperinflammatory states ${ }^{6}$. Many other studies have also documented the correlation of oral health diseases with T2DM. A case control study was done in subjects of T2DM with observations of multiple oral impacts that included; root caries: plaque index: decay, missing and filled teeth index (DMFT): tooth mobility: probing depth and bleeding on probing. Dietary habits constituted a major role for ODIP (oral impact on daily performance) in the patients of diabetes. The consumption of bread and meat in patients of T2DM had a huge influence on ODIP, which was fluctuated according to their glycemic statuses ${ }^{7}$. A cross-sectional observational study showed $42.5 \%$ oral soft tissue lesions and $25 \%$ candidiasis in patients of T2DM, recommending early screening of diabetes in them ${ }^{8}$.

A literature on subjects of T2DM revealed the severity of periodontitis to be more prevalent in males as compared to females, with the exception of cases that were on insulin therapies ${ }^{9}$. A research highlighted a significant association of periodontal diseases with T2DM, showing poor glycemic control and salivary chromogranin A presence as the risk factors for periodontitis development in the associated subjects ${ }^{10}$. Also, a crosssectional study in T2DM non-smokers that were under anti-diabetic drugs, showed poor glycemic controls with moderate to severe periodontitis and better glucose control rates with mild to nil periodontitis. One more survey showed the positive association of T2DM or obesity linked T2DM with periodontitis in Vietnamese patients.

This above mentioned evidence on the association of periodontitis with T2DM is variable and shows multipronged findings. We planned to assess an association between T2DM related dyslipidemia and periodontitis in comparison to patients having T2DM without dyslipidemia. This evidence would highlight the gap in literature for the patients of diabetes with dyslipidemia in our local context. The aim of present study was to correlate the association of periodontal diseases with T2DM and diabetes linked dyslipidemia in Pakistani population, jeopardizing general health.

\section{METHODOLOGY}

This research study was done according to comparative cross-sectional design, using nonprobability, consecutive sampling technique. The duration of study was one year, from January 2018 to January 2019. Subjects for sampling were enrolled from Fauji Foundation Hospital and Pak Emirates Military Hospital, Rawalpindi, Pakistan. Since the study investigations were based at Army Medical College, National University of Medical Sciences (NUMS), the ethical approval was taken from this institution ethics review committee. Freshly diagnosed patients having T2DM and T2DM linked dyslipidemia were included in the study. Sample size was calculated using standardized WHO calculator with following statistical assumptions; confidence level = $95 \%$, alpha error $=5 \%$, study power $=80 \%$, anticipated diabetic population with dental problems $=73.3 \%$ and assumed relative precision of $10 \% 11$. The calculated sample size (N) was 75 subjects. Patients without T2DM but having dyslipidemia, those on lipid lowering drug therapies and those possessing comorbids/chronic illnesses were excluded from the study. Study began with an informed written consent, detailed history, general physical and systemic examinations and proforma (containing personal, lifestyle and health related laboratory tests details). Whole venous blood sample $(5 \mathrm{ml})$ of each patient was drawn in fasting periods. Fasting blood sugar (FBS): $\geq 7.0$ $\mathrm{mmol} / \mathrm{l}$ and random blood sugar (RBS): $>11.1$ $\mathrm{mmol} / \mathrm{l}$ was marked as the cut off values, as per American Diabetes Association (ADA). HbA1c $>6.5 \%$ was considered significant for T2DM diagnosis. Fasting lipid profile encompassed serum TC $>5.20 \mathrm{mmol} / \mathrm{l}$, TAGs $>1.70 \mathrm{mmol} / \mathrm{l}$, LDL-C $>2.50 \mathrm{mmol} / \mathrm{l}$ and HDL-C $<1.0 \mathrm{mmol} / \mathrm{l}$, for tagging patients of diabetes with dyslipidemia. Hepatitis screening included hepatitis B surface antigen (HBsAg) and hepatitis $\mathrm{C}$ antibody (Anti$\mathrm{HCV}$ ) tests. Among many studied parameters of this study, importance of oral health to general well-being of subjects of diabetes emerged as a 
fundamental concern. Oral health parameters in our subjects were divided into two categories; T2DM with periodontitis and T2DM without periodontitis.

Data analysis was carried out using SPSS-21 software. The expression of data for baseline characteristics (age and gender) was executed in terms of frequencies and percentages, and the lipid profile data was expressed as mean \pm standard deviation (SD). Differences between dental categories in T2DM and T2DM with dyslipidemia were analyzed using Pearson's chi-square test. Whereas, the association of baseline characteristics and lipid profile with periodontal diseases was done using independent samples t-test, with consideration of a significant $p$-value of $\leq 0.05$.

\section{RESULTS}

Out of 75 patients, percentage and frequency of males and females were [19 (25.3\%)] and [56 $(74.7 \%)]$, respectively (table-I). The percentage and frequency of age groups were; 21 to 30 years [4 (5.3\%)], 31 to 40 years [5 $(6.7 \%)], 41$ to 50 years [25 (33.3\%)], 51 to 60 years [22 (29.3\%)] and > 61 years [19 $(25.3 \%)]$ (table-I).

Patients of diabetes with dyslipidemia and diabetes without dyslipidemia displayed percentage and frequency of $37(49.3 \%)$ and 38 (50.7\%), respectively (fig-1).

Subjects of diabetes with dyslipidemia showed total cholesterol levels of $5.05 \pm 1.36 \mathrm{mmol} / \mathrm{l}$, triglycerides levels of $2.12 \pm 1.34 \mathrm{mmol} / \mathrm{l}$, low density lipoprotein levels of $2.64 \pm 1.05 \mathrm{mmol} / 1$ and high density lipoprotein levels of $1.02 \pm 0.38$ $\mathrm{mmol} / \mathrm{l}$, respectively (table-II).

Percentage and frequency of males and females with diabetes and periodontitis were $7(25.9 \%)$ and $20(74.1 \%)$, and males and females with diabetes but without periodontitis were 12 $(25 \%)$ and $36(75 \%)$, respectively (table-III). Thus, results revealed that male patients of diabetes, with and without periodontitis 7 (25.9\%) and 12 (25\%) possessed lower numbers than female patients of diabetes, with and without periodontitis $20(74.1 \%)$ and $36(75 \%)$. Patients of diabetes with periodontitis and diabetes without periodontitis had mean ages of [56.33 \pm 10.88 years] and [50.31 \pm 11.14 years], respectively (table-III). The results displayed mean ages with a higher incline towards T2DM with periodontitis group [56.33 \pm 10.88 years], as compared to subjects of T2DM without periodontitis group [50.31 \pm 11.14 years], which showed lower mean ages. T2DM with periodontitis showed total cholesterol levels of $5.41 \pm 1.70 \mathrm{mmol} / \mathrm{l}$, triglycerides levels of 2.55 $\pm 1.46 \mathrm{mmol} / \mathrm{l}$, low density lipoprotein levels of $3.01 \pm 1.22 \mathrm{mmol} / 1$ and high density lipoprotein levels of $0.97 \pm 0.48 \mathrm{mmol} / \mathrm{l}$, respectively (tableIII). T2DM without periodontitis showed total

Table-I: The baseline characteristics (age and gender) of study subjects $(n=75)$.

\begin{tabular}{l|l|c}
\hline \multirow{2}{*}{ Parameters } & Male & n (\%) \\
\cline { 2 - 3 } & Female & $19(25.3)$ \\
\hline \multirow{4}{*}{ Age (Years) } & 21 to 30 & $56(74.7)$ \\
\cline { 2 - 3 } & 31 to 40 & $4(5.3)$ \\
\cline { 2 - 3 } & 41 to 50 & $5(6.7)$ \\
\cline { 2 - 3 } & 51 to 60 & $25(33.3)$ \\
\cline { 2 - 3 } & 61 and above & $19(29.3)$ \\
\hline
\end{tabular}

Table-II: The status of lipid profile characteristics of study subjects $(n=75)$.

\begin{tabular}{l|c}
\hline Parameters & Mean \pm SD \\
\hline Total Serum Cholesterol $(\mathrm{mmol} / \mathrm{l})$ & $5.05 \pm 1.36$ \\
\hline Serum Triglycerides $(\mathrm{mmol} / \mathrm{l})$ & $2.12 \pm 1.34$ \\
\hline $\begin{array}{l}\text { Serum Low Density Lipoprotein } \\
\text { Cholesterol (mmol/l) }\end{array}$ & $2.64 \pm 1.05$ \\
\hline $\begin{array}{l}\text { Serum High Density Lipoprotein } \\
\text { Cholesterol (mmol/l) }\end{array}$ & $1.02 \pm 0.38$
\end{tabular}

cholesterol levels of $4.84 \pm 1.09 \mathrm{mmol} / \mathrm{l}$, triglycerides levels of $1.88 \pm 1.22 \mathrm{mmol} / \mathrm{l}$, low density lipoprotein levels of $2.43 \pm 0.89 \mathrm{mmol} / 1$ and high density lipoprotein levels of $1.04 \pm 0.32 \mathrm{mmol} / \mathrm{l}$, respectively (table-III). Therefore, results concluded that subjects of T2DM with periodontitis showed marked lipid profile derangements as compared to the subjects of T2DM without periodontitis. Total cholesterol, low density lipoproteins and high density lipoproteins were found raised in subjects of T2DM with periodontitis [5.41 \pm $1.70 \mathrm{mmol} / \mathrm{l}],[3.01 \pm 1.22 \mathrm{mmol} / \mathrm{l}]$ and [0.97 \pm $0.48 \mathrm{mmol} / \mathrm{l}]$, when compared to the subjects of T2DM without periodontitis group $[4.84 \pm 1.09$ 
$\mathrm{mmol} / \mathrm{l}, 2.43 \pm 0.89 \mathrm{mmol} / \mathrm{l}$ and $1.04 \pm 0.32 \mathrm{mmol}$ /1] that displayed no lipid profile derangements. However, triglycerides value in subjects of T2DM with periodontitis $[2.55 \pm 1.46 \mathrm{mmol} / \mathrm{l}]$ was dyslipidemia and periodontitis [16 (59.3\%)] were greater in number, when contrasted with subjects of T2DM without dyslipidemia and periodontitis [27 (56.3\%)], subjects of T2DM with

Table-III: The association of baseline and lipid profile characteristics of study subjects with periodontal diseases.

\begin{tabular}{|c|c|c|c|c|}
\hline \multirow{2}{*}{\multicolumn{2}{|c|}{ Parameters }} & \multicolumn{2}{|c|}{ Oral Health Parameters } & \multirow{4}{*}{$\begin{array}{c}\begin{array}{c}p- \\
\text { value }\end{array} \\
0.929\end{array}$} \\
\hline & & $\begin{array}{c}\text { Group I } \\
\text { Type } 2 \text { Diabetes Mellitus } \\
\text { with Periodontitis }(n=27)\end{array}$ & $\begin{array}{c}\text { Group II } \\
\text { Type } 2 \text { Diabetes Mellitus } \\
\text { Without Periodontitis }(n=48)\end{array}$ & \\
\hline \multirow{2}{*}{ Gender } & Male & $7(25.9 \%)$ & $12(25 \%)$ & \\
\hline & Female & $20(74.1 \%)$ & $36(75 \%)$ & \\
\hline \multicolumn{2}{|c|}{ Age (Years) } & $56.33 \pm 10.88$ & $50.31 \pm 11.14$ & 0.027 \\
\hline \multirow{4}{*}{$\begin{array}{l}\text { Lipid } \\
\text { Profile }\end{array}$} & Total Serum Cholesterol (mmol/l) & $5.41 \pm 1.70$ & $4.84 \pm 1.09$ & 0.082 \\
\hline & Serum Triglycerides $(\mathrm{mmol} / \mathrm{l})$ & $2.55 \pm 1.46$ & $1.88 \pm 1.22$ & 0.037 \\
\hline & $\begin{array}{l}\text { Serum Low Density Lipoprotein } \\
\text { Cholesterol }(\mathrm{mmol} / \mathrm{l})\end{array}$ & $3.01 \pm 1.22$ & $2.43 \pm 0.89$ & 0.022 \\
\hline & $\begin{array}{l}\text { Serum High Density Lipoprotein } \\
\text { Cholesterol }(\mathrm{mmol} / \mathrm{l})\end{array}$ & $0.97 \pm 0.48$ & $1.04 \pm 0.32$ & 0.437 \\
\hline \multirow{2}{*}{$\begin{array}{l}\text { Type } 2 \\
\text { Diabetes } \\
\text { Catego- } \\
\text { ries }\end{array}$} & $\begin{array}{l}\text { Type } 2 \text { Diabetes Mellitus With } \\
\text { Dyslipidemia }\end{array}$ & $16(59.3 \%)$ & $21(43.8 \%)$ & \multirow{2}{*}{0.197} \\
\hline & $\begin{array}{l}\text { Type } 2 \text { Diabetes Mellitus Without } \\
\text { Dyslipidemia }\end{array}$ & $11(40.7 \%)$ & $27(56.3 \%)$ & \\
\hline
\end{tabular}

pictured with marked abnormalities, as compared to the subjects of T2DM without periodontitis $[1.88 \pm 1.22 \mathrm{mmol} / \mathrm{l}]$ that showed mild alterations. Percentage and frequency of T2DM with dyslipidemia and periodontitis were $16(59.3 \%)$,

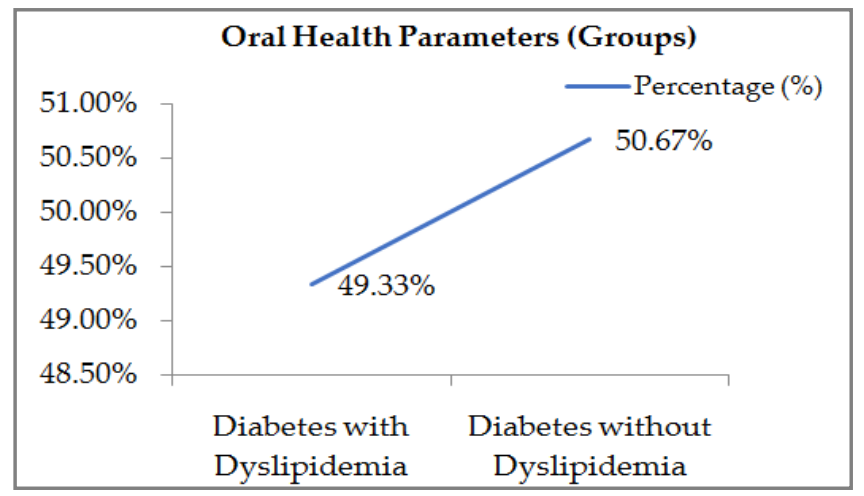

Figure-1: General distribution of study subjects possessing type 2 diabetes mellitus with dyslipidemia and type 2 diabetes mellitus without dyslipidemia.

T2DM with dyslipidemia and without periodontitis were 21 (43.8\%), T2DM without dyslipidemia but with periodontitis were $11(40.7 \%)$, and T2 DM without dyslipidemia and periodontitis were $27(56.3 \%)$, respectively (table-III). Hence, it was concluded that the subjects of T2DM with dyslipidemia and without periodontitis [21 $(43.8 \%)]$, and subjects of T2DM without dyslipidemia but with periodontitis [11 (40.7\%)].

\section{DISCUSSION}

The current study proves significant derangement in the lipid profiles of patients presenting with coexistent T2DM and periodontal diseases, with affected number of cases to be $16(59.3 \%)$. Age was also seen momentous in this research analysis, highlighting middle aged females to be influenced vastly, followed by middle to late aged males, for the prevalence of T2DM linked dyslipidemias and periodontitis. Diabetes mellitus is seen about 1 in every $11^{\text {th }}$ adult and documented as the ninth major cause for worldwide mortalities that has affected global population in previous three decades up to four folds. The T2DM, also known as adult-onset diabetes is recorded to affect Asia frequently and making it the epicenter for the global T2DM epidemic ${ }^{12}$.

Dyslipidemia is considered as a major risk factor for triggering cardiovascular events (CVDs) in T2DM, leading to marked morbidities and mortalities in male and female subjects ${ }^{13}$. 
Whereas, majority of the subjects of T2DM may carry certain additional concerns to CVDs, such as periodontitis. Periodontitis is an enduring infectious and inflammatory disorder, occurring due to sub gingival build-up of plaques and specific periodontopathogenic bacterial actions and it is seen three times superior in the cases of T2DM. Positive associations have been illustrious between the severity of periodontitis and degree of hyperglycemia ${ }^{14}$. Periodontitis has also proven its severity connections with CVD in subjects of T2DM. In order to ameliorate the life quality of subjects of T2DM suffering with CVDs and periodontitis, and impediment of their medical expense burden, nonsurgical periodontal treatment (root planning and scaling) is a requisite, along with life style modifications (healthy diet, healthy body weight maintenance and staying physically active).

The T2DM has been reported in previous literatures, such as in the WHO fact sheet of DM 2016 for Pakistan by Ms Laura Sminkey, to affect both the genders ${ }^{4}$. The DM prevalence is $17.15 \%$ in rural and $22.04 \%$ in urban areas of the country. The Khyber Pakhtun Khwa state showed prevalence rate of $11.1 \%$, while Balochistan state uncovered the prevalence rate of $10.8 \%$ for diabetes in both sexes. Whereas, Punjab state revealed prevalence rate of diabetes in males as $16.6 \%$ and in females as $19.3 \%$. On the other hand, Sindh state highlighted prevalence rate of $16.2 \%$ in males and $11.7 \%$ in females ${ }^{15}$. Some works such as diabetes fact sheets in Korea 2018 by Jong Chul Won, focused on males and females to be affected equally with T2DM, and with higher ascendancy towards increased ages, up to 60 years in men and 70 years in women ${ }^{16}$. Our findings included both males and females as well, but there were fewer males targeted as compared to females in the stu$\mathrm{dy}$, hence it was difficult to conclude that males were least prevalent than females for T2DM.

Past studies done by Sulaiman Nabil in subjects for early detection of T2DM in United Arab Emirates showed age groups between 40 to 60 years to be mostly influenced for the occurrence of T2DM, with early onset of dyslipidemias in young adults ${ }^{17}$. Our findings also showed middle aged males who suffered from T2DM linked dyslipidemia to be less predominant than females. Also, young (< 40 years) and older (> 61 years) ages were little prevalent for the onset of T2DM in our survey, that was quite similar to many former work results. Past literatures, such as studies conducted in Karachi, Pakistan by Sehrish Shafique in 2019, have shown positive association of T2DM with dyslipidemia in males affecting old ages. Out of targeted 383 patients of T2DM, 210 patients accompanied dyslipidemia ${ }^{18}$. Also, late ages (> 50 years in obese and $\leq 45$ in non-obese) females were seen frequently suffering from T2DM without dyslipidemia in a research conducted at Beijing by Jiajia Jiang in 2019, while determining the association between age at natural menopause and risk of T2DM in postmenopausal women with and without obesity ${ }^{19}$. These prior study results appeared similar to our outcomes, which also presented T2DM males with dyslipidemia and T2DM females without dyslipidemia to be more endemic in late ages. The lipid profile derangements in T2DM with dyslipidemia have been seen followed by many mixed patterns. One similar study was conducted in Pakistan by Fauzia Jan in 2018 on patients of T2DM showed alteration in the lipid profile parameters (i.e. positive association between FBS and TC, TAGs and LDL-C and negative correlation between FBS and HDL-C) ${ }^{20}$. In our outcomes, serum triglycerides and LDL-C levels in patients of T2DM with dyslipidemia displayed marked derangements, while total cholesterol and HDL-C levels exhibited normal picture.

Dental problems in our subjects included dental caries, artificial teeth, swollen and painful gums, tooth losses, plaques and root canal treatments. Previous reports on T2DM linked with dyslipidemia and periodontitis have been documented with striking changes in the TC, TAGs and LDL-C values. Thus, positive ties have been found between periodontitis and T2DM with dyslipidemia (correlation of pro-inflammatory cytokines with worsened glycemic, lipid and periodontal parameters), affecting middle to late 
ages in a research conducted by Rafael Nepomuceno in 2019 at Brazil. Lipid derangements documented were high TC, LDL-C and TAGs levels ${ }^{6}$. Similar lipid profile modifications were seen in our subjects of T2DM with periodontitis as well, which included raised TC, TAGs, LDL-C with addition of diminished HDL-C values. While, our subjects of T2DM without periodontitis showed near normal lipid profile parameters with exception of minute changes in TAGs (mildly raised) only. Thus, it was concluded that subjects of T2DM with periodontitis showed marked changes in the lipid profile, when contrasted to subjects of T2DM without periodontitis.

The T2DM with dyslipidemia has been evidenced to directly associate with active periodontitis (1.20 times higher risk) less distinctly in males as compared to females, affecting late ages, followed by the middle ages, in a Korean study by Jae-Hong in 201921. However, this present study conclusion disclosed that middle to late aged males were led by middle aged females for T2DM linked dyslipidemia and periodontitis. And our cases of T2DM without periodontitis also exposed males in lesser records than females. Hence, determined that females who suffered from T2DM associated periodontitis were more dominant than males of the same group and also were more prevalent than females and males of T2DM without periodontitis group.

Preceding researches, one such directed by Baochen et al in 2019 at United States of America, has documented mean ages of subjects of T2DM with periodontitis to be around $51.5 \pm 2.9$ years ${ }^{22}$. This nearly parallels with our findings too, which displayed mean age of $56.33 \pm 10.88$ years to be more highlighted in the subjects of T2DM with periodontitis. Whereas, subjects of T2DM without periodontitis showed mean age around $50.31 \pm$ 11.14 years, which relates with older investigates as well that displayed middle ages to be more affected in cases of T2DM without periodontitis. Thus, it could be drawn from our study conclusions that higher leaning was seen in mean ages of T2DM linked with periodontitis group as compared to T2DM linked without periodontitis group.

Many former revisions have displayed strong correlation of T2DM with periodontitis and risk of dyslipidemia, accompanied with poor glycemic controls in the affiliated subjects, such as a cross-sectional study on T2DM non-smokers, under anti-diabetic drugs, investigated by Zuila Albuquerque in 2018 at urban area of Sobral, Ceara, Brazil. Subjects with no or mild periodontitis showed FBS levels of $6.8 \pm 2.0 \mathrm{mmol} / \mathrm{l}$, as compared to the subjects with moderate to severe periodontitis that displayed FBS levels of $7.6 \pm$ $1.9 \mathrm{mmol} / \mathrm{l}^{23}$. Our results correlated with these earlier literatures, showing relationship of T2DM with dyslipidemia and periodontitis to be more prevalent and resilient, as compared to T2DM without dyslipidemia and periodontitis, T2DM with dyslipidemia but without periodontitis, and T2DM without dyslipidemia but with periodontitis. Thus, periodontitis has been documented to impart dominant role in its association with T2DM linked and non-linked dyslipidemia. Our results also correlated with multiple former studies, implicating the medical significance of periodontitis as a risk indicator for T2DM.

\section{RECOMMENDATION}

Good glycemic control maintenances and healthy lifestyle modifications can prevent the development of diabetes linked micro and macrovascular complications, and consequently periodontitis too. Also, further epidemiological and molecular researches will be required to ascertain the risk factors responsible for triggering periodontitis linked diabetic dyslipidemia.

\section{ACKNOWLEDGEMENT}

We are thankful to the department of Biochemistry \& Molecular Biology, Army Medical College, National University of Medical Sciences (NUMS), for supporting the research.

\section{Funding Source}

The research survey was funded by the National University of Medical Sciences (NUMS), Rawalpindi, Pakistan. 


\section{CONCLUSION}

It is concluded that there was an overall significant association between type 2 diabetes mellitus related dyslipidemia and periodontitis. Further, there was significant difference in age and gender of the study subjects, as we found middle to late aged males to be less affected, whereas middle aged females were more often affected.

\section{CONFLICT OF INTEREST}

This study has no conflict of interest to be declared by any author.

\section{REFERENCES}

1. Unthakee Z, Goldenberg R, Katz P. Definition, classification and diagnosis of diabetes, prediabetes and metabolic syndrome. Can J Diabetes 2018; 42(Suppl-1): S10-15.

2. Shestakova MV, Dedov II. Diabetes mellitus in the Russian Federation: Arguments and facts. Ter Arkh 2016; 88(10): 4-8.

3. IDF releases report of global survey on access to medicines and supplies for people with diabetes. Diabetes Res Clin Pract 2017; 129(1): 224-25.

4. minkey ML. World Health Organization - Diabetes country profiles, 2016. Available from: http://www.who.int

5. Rehan M, Waqar S, Khan H. Prevalence of dyslipidemia among diabetic patients in a tertiary care Hospital. J Islamabad Med Dent College 2018; 7(2): 136-39.

6. Nepomuceno R, Vallerini BD, da Silva RL, Corbi SC, Bastos AD, dos Santos RA, et al. Systemic expression of genes related to inflammation and lipid metabolism in patients with dyslipidemia, type 2 diabetes mellitus and chronic periodontitis. Diabetes Metab Syndr 2019; 13(4): 2715-22.

7. Mohamed HG, Mustafa K, Ibrahim SO, Åstrøm AN. Dietary habits, oral impact on daily performance and type 2 diabetes: a matched case-control study from Sudan. Health Qual Life Outcomes 2017; 15(1): 111.

8. Negi M, Holmes H, Stephen LX. Diabetic status of patients presenting for dental treatment. S Afr Dent J 2018; 73(4): 274-77.

9. Schulze A, Busse M. Gender differences in periodontal status and oral hygiene of non-diabetic and type 2 diabetic patients. Open Dent J 2016; 10(1): 287-97.

10. Kogawa EM, Grisi DC, Falcão DP, Amorim IA, Rezende TM, da
Silva IC, et al. Impact of glycemic control on oral health status in type 2 diabetes individuals and its association with salivary and plasma levels of chromogranin A. Arch Oral Biol 2016; 62(1): 10-19.

11. Malvania EA, Sheth SA, Sharma AS, Mansuri S, Shaikh F, Sahani S. Dental caries prevalence among type II diabetic and nondiabetic adults attending a hospital. J Int Soc Prev Community Dent 2016; 6(Suppl-3): S232-36.

12. Zheng Y, Ley SH, Hu FB. Global aetiology and epidemiology of type 2 diabetes mellitus and its complications. Nat Rev Endocrinol 2018; 14(2): 88-98.

13. Feingold KR, Grunfeld C. Diabetes and dyslipidemia. In Endo text [Internet] 2019. Avalable from: MDText.com, Inc.

14. Agarwal S, Gangaram D, Saleem M. Periodontitis in patients with type 2 diabetes mellitus. Ann Int Med Den Res 2018; 4(3): 58-65.

15. Hussain A, Ali I. Diabetes mellitus in Pakistan: A major public health concern. Arch Pharm Pract 2016; 7(1): 30-33.

16. Kim BY, Won JC, Lee JH, Kim HS, Park JH, Ha KH, et al. Diabetes Fact Sheets in Korea, 2018: an appraisal of current status. Diabetes Metab 2019; 43(4): 487-94.

17. Sulaiman N, Mahmoud I, Hussein A, Elbadawi S, Abusnana S, Zimmet P, et al. Diabetes risk score in the United Arab Emirates: a screening tool for the early detection of type 2 diabetes mellitus. BMJ Open Diabetes Res Care 2018; 6(1): 1-5.

18. Shafique S, Mirza DSS, Tabassum S, Faraz N. Frequency of dyslipidemia in type 2 diabetic patients in Karachi. J Bahria Uni Med Dental Coll 2019; 9(2): 133-36.

19. Jiang J, Cui J, Wang A, Mu Y, Yan Y, Liu F, et al. Association between age at natural menopause and risk of type 2 diabetes in postmenopausal women with and without obesity. J Clin Endocrinol Metab 2019; 104(7): 3039-48.

20. Jan F, Saeed M, Zia S, Rahman R, Muzaffar S, Waheed A. Type 2 Diabetes mellitus; association of dyslipidemia and magnesium levels in type 2 diabetes mellitus. Professional Med J 2018; 25(12): 1972-78

21. Lee JH, Kim DH, Nam BR, Jeong SN. Association between diabetes mellitus and active periodontitis in Korean adults from Korea National Health and Nutrition Examination Surveys 2010-2015. Oral Biol Res 2019; 43(3): 202-09.

22. Shi B, Lux R, Klokkevold P, Chang M, Barnard E, Haake S, et al. The subgingival microbiome associated with periodontitis in type 2 diabetes mellitus. Microb Ecol 2020; 14(1): 519-30.

23. Taboza ZA, Costa KL, Silveira VR, Furlaneto FA, Montenegro R, Russell S, et al. Periodontitis, edentulism and glycemic control in patients with type 2 diabetes: a cross-sectional study. BMJ Open Diabetes Res Care 2018; 6(1): 1-5. 